\title{
Foreword: Challenging views of Alzheimer's disease - 2004
}

\author{
J. Wesson Ashford ${ }^{\mathrm{a}}$, Keith A. Crutcher ${ }^{\mathrm{b}}$, Stephen R. Robinson ${ }^{\mathrm{c}}$ and Mark A. Smith ${ }^{\mathrm{d}}$ \\ ${ }^{a}$ Stanford/VA Alzheimer Center, Palo Alto, California, USA \\ ${ }^{\mathrm{b}}$ University of Cincinnati, Cincinnati, Ohio, USA \\ ${ }^{\mathrm{c}}$ Monash University, Monash, Australia \\ ${ }^{\mathrm{d}}$ Case Western Reserve University, Cleveland, Ohio, USA
}

In this, and forthcoming issues of the Journal of Alzheimer's Disease (JAD), it is our privilege to communicate summaries of the debates held at the recent conference, Challenging Views of Alzheimer's Disease - 2004, that was held in conjunction with the International Conference on Alzheimer's Disease and Related Disorders in Philadelphia (July 2004).

The Challenging Views of Alzheimer's Disease series of meetings offers a unique format where controversy and debate hold center stage - egos and rigidthinking are left outdoors. The goal is to challenge established hypotheses and dogma in an effort to better understand disease pathogenesis, diagnosis and treat- ment. We are truly grateful to those scientists and clinicians who rose admirably to the challenge, to our sponsors (Voyager Pharmaceutical Corporation, The Wild Blueberry Association of North America, Philadelphia College of Osteopathic Medicine, Alzheimer's Association, Cephalon, and the Alzheimer Research Forum), and to the Editor-in-Chief of the JAD, Dr. George Perry, for providing the entire Alzheimer community the open and transparent environment necessary to promote novel thinking. We hope that you will enjoy the synopses provided in this and upcoming issues of JAD and that this will compel you to join us at our upcoming meetings in Sorrento in 2005 and Madrid in 2006. 